\title{
Exome-wide analysis of mutational burden in patients with typical and atypical Rolandic epilepsy
}

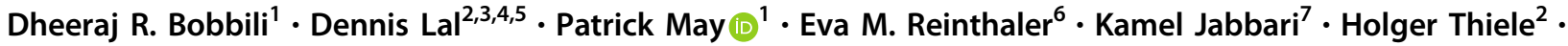 \\ Michael Nothnagel ${ }^{2}$. Wiktor Jurkowski ${ }^{1,8} \cdot$ Martha Feucht $^{9} \cdot$ Peter Nürnberg ${ }^{2} \cdot$ Holger Lerche $^{10} \cdot$ Fritz Zimprich $^{6}$. \\ Roland Krause $^{1} \cdot$ Bernd A. Neubauer $^{11}$ - Eva M. Reinthaler ${ }^{6}$. Fritz Zimprich $^{6}$ - Martha Feucht ${ }^{12}$. \\ Hannelore Steinböck ${ }^{13}$ - Birgit Neophytou ${ }^{14}$. Julia Geldner ${ }^{15}$. Ursula Gruber-Sedlmayr ${ }^{16}$. Edda Haberlandt ${ }^{17}$. \\ Gabriel M. Ronen ${ }^{18} \cdot$ Janine Altmüller ${ }^{2} \cdot$ Dennis Lal ${ }^{2} \cdot$ Peter Nürnberg ${ }^{2} \cdot$ Thomas Sander $^{2} \cdot$ Holger Thiele $^{2}$. \\ Roland Krause $^{1} \cdot$ Patrick May $^{1} \cdot$ Rudi Balling ${ }^{1} \cdot$ Holger Lerche $^{10} \cdot$ Bernd A. Neubauer $^{11}$ \\ EUROEPINOMICS COGIE Consortium
}

Received: 8 May 2017 / Revised: 27 September 2017 / Accepted: 6 October 2017 / Published online: 22 January 2018

(c) European Society of Human Genetics 2018

\begin{abstract}
Rolandic epilepsy (RE) is the most common focal epilepsy in childhood. To date no hypothesis-free exome-wide mutational screen has been conducted for RE and atypical RE (ARE). Here we report on whole-exome sequencing of 194 unrelated patients with RE/ARE and 567 ethnically matched population controls. We identified an exome-wide significantly enriched burden for deleterious and loss-of-function variants only for the established RE/ARE gene GRIN2A. The statistical significance of the enrichment disappeared after removing ARE patients. For several disease-related gene-sets, an odds ratio $>1$ was detected for loss-of-function variants.
\end{abstract}

\section{Introduction}

Rolandic epilepsy (RE), or epilepsy with centro-temporal spikes (CTS), is one of the most common epilepsy syndromes of childhood. RE is related to rarer and less benign epilepsy syndromes, including atypical benign partial epilepsy, Landau-Kleffner syndrome and epileptic encephalopathy with continuous spike-and-waves during sleep, referred to as RE-related syndromes or atypical rolandic epilepsy (ARE) [1]. In up to $20 \%$ sib pairs or

EuroEPINOMICS consortium contributing partners are listed above References.

D.R. Bobbili, D. Lal and P. May contributed equally to this work.

Electronic supplementary material The online version of this article (https://doi.org/10.1038/s41431-017-0034-x) contains supplementary material, which is available to authorized users.

Roland Krause

roland.krause@uni.lu

$\triangle$ Bernd A. Neubauer

Bernd.A.Neubauer@paediat.med.uni-giessen.de

Extended author information available on the last page of the article families, mutations affecting GRIN2A, a subunit of the excitatory glutamate receptor $N$-methyl-D-aspartate (NMDA), were found implicated as major risk factor for RE and ARE by us and others [2,3]. Recently, the association of the genes RBFOX1, RBFOX3, DEPDC5, GABRG2 and genomic duplications at $16 \mathrm{p} 11.2$ in $1.5-2.0 \%$ was identified in patients with RE and ARE [4-6] through candidate gene and loci screens. In the current study, an unbiased exomewide survey was conducted in the RE/ARE cohort.

\section{Patients and methods}

\section{Study participants}

Two hundred and four unrelated European Rolandic cases (182 RE, 22 ARE) and 728 population control subjects were included [6]. Children with (typical) RE suffer from perisylvian oromotor seizures frequently starting during sleep. In adolescence, the epilepsy resolves spontaneously, frequently without any intellectual sequels. ARE share the essential electroencephalography feature with RE but show a different seizure symptomatology by their own or in addition to rolandic seizures. Seizures, like in RE, resolve 
spontaneously, but cognitive outcome is guarded in ARE. In detail, these epilepsies are: atypical benign partial epilepsy of childhood, with atonic seizures and atypical absences in addition to rolandic seizures; Landau-Kleffner syndrome, with loss of speech and cognitive decline; and epilepsia-aphasia syndrome with seizures and language dysfunction [1, 6]. Written informed consent was obtained from participating subjects and, if appropriate, from both patients and adolescents.

\section{Data generation and processing}

Exome sequencing of all individuals was performed with the Illumina HiSeq 2000 using the EZ Human Exome Library Kit (NimbleGen, Madison, WI). Sequencing adapters were trimmed and samples with $<30 \times$ mean depth or $<70 \%$ total exome coverage at $20 \times$ mean depth of coverage were excluded from further analysis. Variant calling was performed in targeted exonic intervals with $100 \mathrm{bp}$ padding using the GATK best practices pipeline [7] against the GRCh37 human reference genome followed by multi-allelic variant decomposition and left normalization. Samples were excluded from further analysis if they (i) were not ethnically matched, (ii) were related, (iii) showed discrepancy with reported sex, (iv) had an excess heterozygosity $>3$ SD in any of the quality metrics (NALT, NMIN, NHET, NVAR, RATE and SINGLETON statistics as calculated by PLINKseq istats parameter [8]. The genotypes of variants with read depth $<10$ or genotype quality $<20$ were set to missing. Variants were excluded if they (i) failed variant quality score recalibration (VQSR) or GATK recommended hard filter, (ii) showed missingness $>3 \%$, (iii) were present in repeat regions or (iv) had an average read depth $<10$ in either cases or controls. The ExAC variants were restricted to the exonic intervals used for variant calling in this study, not present in the repeat regions and passed the VQSR threshold.

\section{Variant annotation and filtering}

Variants were annotated using ANNOVAR [9] version 2015 Mar 22 with RefSeq and Ensembl, Combined Annotation Dependent Depletion (CADD) scores [10], allele frequencies and dbNSFP (v3.0) annotations. The samples used in this study are of Non-Finnish European (NFE) ancestry, hence to investigate rare variants, we selected variants having a minor allele frequency $<0.005$ in the European populations of the 1000 genomes, Exome Variant Server and the NFE data from ExAC. We generated three classes of variants for further analyses: (1) deleterious variants (CADD15), which were defined as missense variants with a CADD Phred score $>15$ as it is the median value across all missense and canonical splice site variants [10], (2) loss-offunction (LOF) variants comprising all rare indels, stop gain, stop loss and splice site variants ( 2 nt plus/minus the exon boundary), (3) CADD15+LOF variants as the union of the above two datasets, and (4) rare synonymous variants.

\section{Single variant and gene association analysis}

For the statistical analysis, we employed two independent control cohorts (available in-house and ExAC) to increase reliability and power of the statistical tests. For single variant burden analysis, we applied the single score method in RVTESTS [11] to cases and in-house controls, for which individual genotypes were available. For gene burden analysis, a $2 \times 2$ contingency table was constructed by counting the number of alternate allele counts per gene in patients vs. controls (in-house controls and NFE ExAC controls). We then obtained a one-sided $p$-value, odds ratios and the $95 \%$ confidence intervals [12] by using Fisher's exact test. Resulting $p$ values were corrected for 18,668 RefSeq protein-coding genes [13] by Bonferroni approach. Finally, to ensure the exclusion of false positive association results and following the 'rare variant of large effect hypothesis', we selected those genes that are present in the first quartile of the Residual Variant Intolerance Score (RVIS) distribution [14].

\section{Selection of gene-sets}

We investigated the following four neuron-related gene-sets: (1) genes encoding proteins at synapses downloaded from the SynaptomeDB [15] database ("SYNAPTIC_GENES", $N=$ 1887), (2) genes of postsynaptic signalling complexes including NMDA receptors (NMDARs) and the neuronal activity-regulated cytoskeleton-associated protein (ARC) [16] ("NMDAR_ARC_COMPLEX", $N=80$ ), (3) genes encoding proteins at the inhibitory synapses ("INHIBITORY", $N=$ 5941) and excitatory synapses ("EXCITATORY", $N=5261$ ) [17], and (4) glutamate receptor subunit encoding genes ("GLUTAMATE_RECEPTORS", $N=18$ ). In addition, we included five gene-sets associated with disease and/or mutational intolerance: (1) genes encoding targets of Fragile-XMental-Retardation-1-Protein [18] ("FMRP_TARGETS_DARNELL", $N=1772$ ), (2) genes intolerant for variants from ExAC (“EXAC_CONSTRAINED_GENES", $N=3230$ ), (3) genes intolerant for loss-of-function variants [19] ('constrained') ("CONSTRAINED_GENES_SAMOCHA", $N=$ 1004), (4) a curated list of dominant genes associated with developmental delay obtained from the DECIPHER database [20] ("DDG2P_MONOALLELIC", $N=299$ ), and (5) genes found related before to epileptic encephalopathies [21] ("EPILEPTIC_ENCEPHALOPATHY", $N=73$ ). As control data sets, we used (1) for each dataset the corresponding set of synonymous variants, and (2) the 'non-constraint' gene-set including RefSeq genes that have been found tolerant to LOF variants ("GENES_WITHOUT_CONSTRAINT", $\quad N=$ 
14,417). GRIN2A, as the most significant single gene from the burden analysis, was excluded from all gene-sets in order to test if other genes also contribute to the disease association.

\section{Data availability}

All the CADD15+LOF variants from our study within the "EPILEPTIC_ENCEPHALOPATHY" gene-set were deposited in the Leiden Open Variation Database (LOVD) (https:// databases.lovd.nl/shared/genes). The accession numbers of the deposited variants in LOVD are 188117-188549. Also, the variants present in the cases within the "EPILEPTIC_ENCEPHALOPATHY" gene-set are available in the ClinVar database (https://www.ncbi.nlm.nih.gov/clinvar/) with the accession numbers SCV000588243-SCV000588353. The variants that were described in our previous studies are indicated in Supplementry Table 1.

\section{Gene-set association analysis}

The gene-set association analysis for the different types of variants was performed by using a logistic regression approach using $\mathrm{R}$ (version 3.2) and adjusting for the following confounding variables: the total number of called

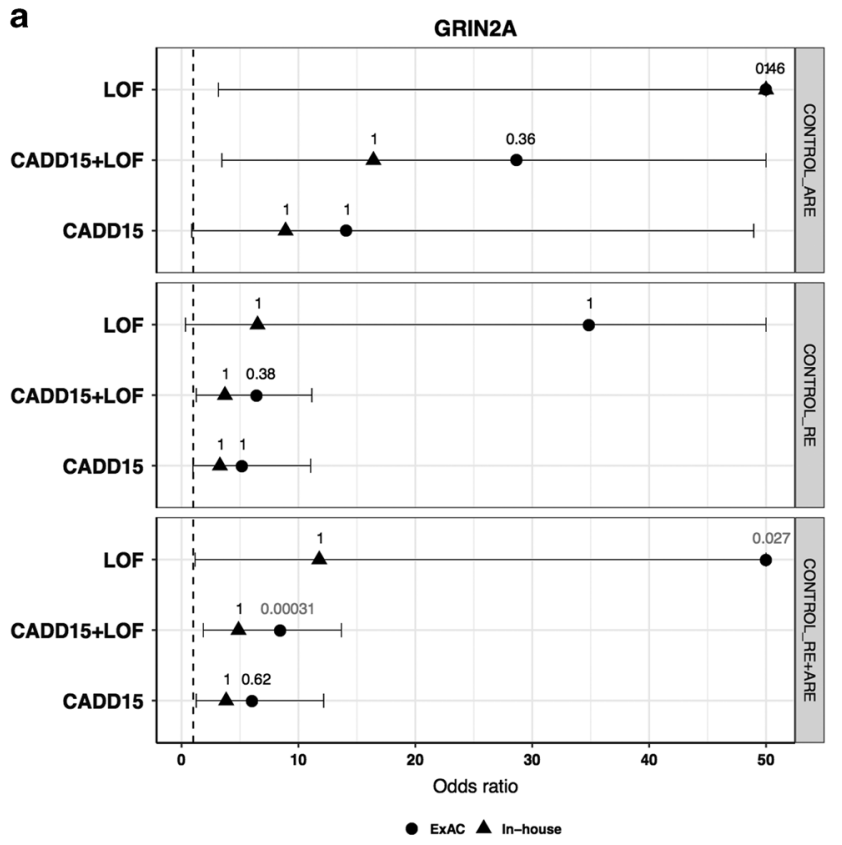

Fig. 1 Burden analysis. Typical Rolandic epilepsy is represented as $\mathrm{RE}$, atypical rolandic epilepsy as ARE and RE plus ARE as ROLANDIC. On the $x$ axis, the odds ratios in cases vs controls are given. The names of the variant classes are given on the $y$ axis. Each panel represents a different dataset. The dashed vertical line represents the expected odds ratio of 1 . The horizontal lines indicate $95 \%$ confidence intervals. a Assessment of risk for deleterious variants in GRIN2A against two control groups (ExAC and In-house). The values genotypes per sample, the total number of rare coding variants per sample, the total number of rare coding singletons (variants observed only once in the entire dataset) per sample, calculated sex, the first four principal components, and the total number of variants per sample for each variant class.

\section{Results}

\section{Exome sequencing and variant filtering}

We performed whole-exome sequencing on 204 patients with RE/ARE and 728 population controls. After quality control, the final dataset consisted of 19 ARE, $175 \mathrm{RE}$ and 567 control samples. From the total of 761 samples, 226,521 exonic and splice site variants were called. The mean transition/transversion ratio equalled 3.39 per sample. After the final filtering 45,881 CADD15, 10,326 LOF and 38,802 synonymous variants were analysed.

\section{Association analysis}

To investigate the mutational burden within the RE spectrum, all associations were assessed for both RE and ARE

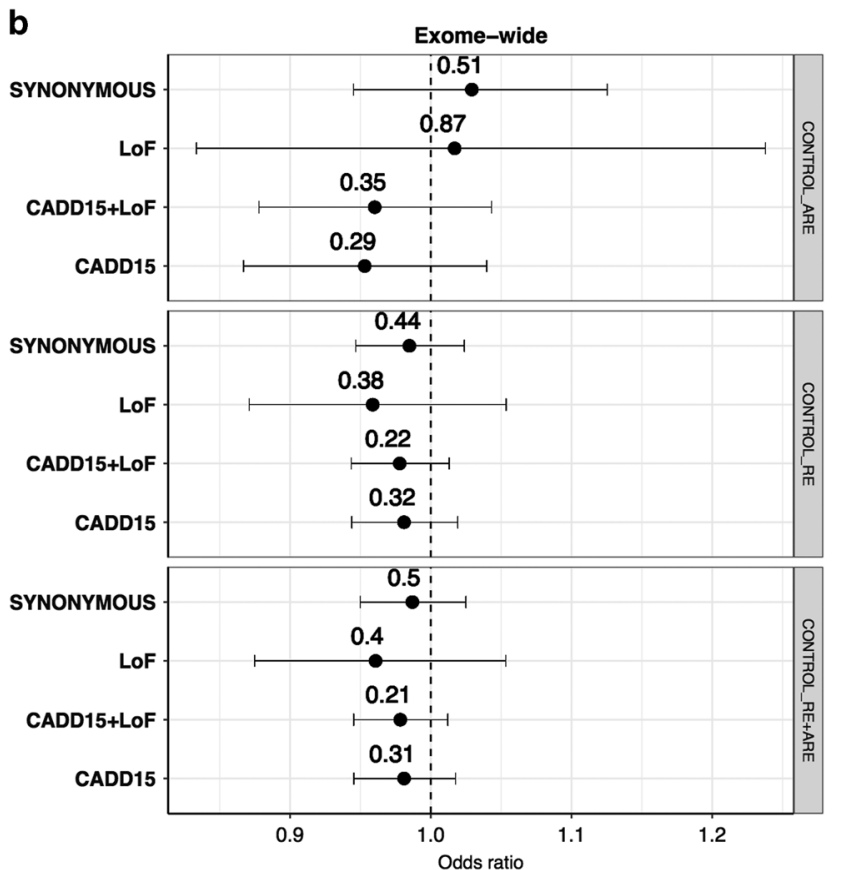

on top of each point represent multiple-testing corrected $p$-values, the ones in red are significant $p$-values and the ones in black are the $p$ values that are not significant after multiple-testing correction. The odds ratios are restricted to a maximum value of 50. b Exome-wide burden analysis by different variant classes. The values on top of each point represent the $p$-value. Synonymous variants serve as a control functional group (colour figure online) 
separately and by combining cases from both phenotypes while assuming them to be a single disease. In comparison to 567 in-house controls, we did not observe statistically significant burden in any of the variants or genes in cases after multiple-testing correction. In order to increase the statistical power, we used the non-Finnish European (NFE) ExAC cohort as an additional control dataset. Association testing against the much larger NFE-ExAC cohort $(N=$ 33,370 ) identified an exome-wide significant burden for CADD15, CADD15+LOF and LOF variants for GRIN2A
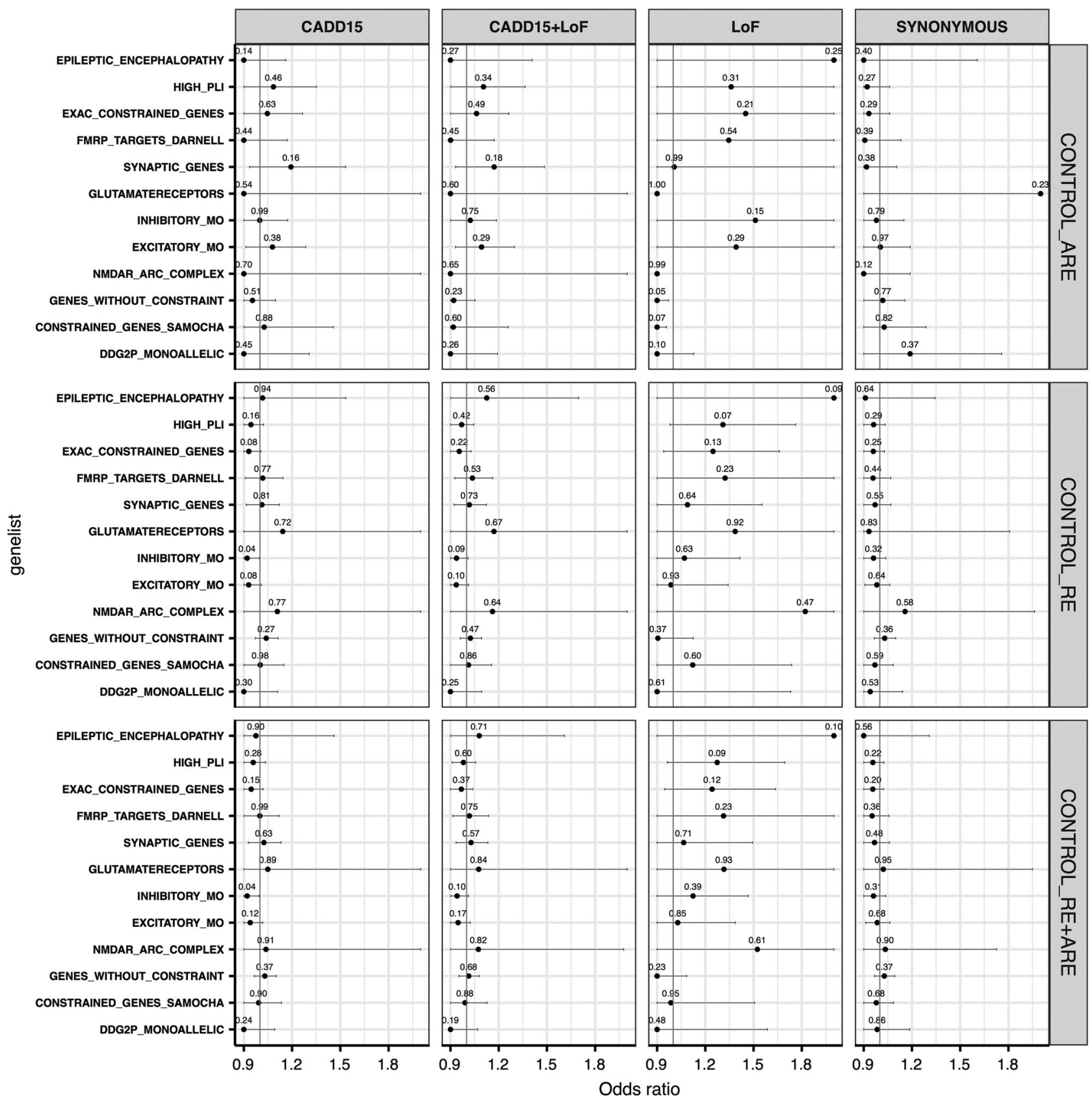

Fig. 2 Gene-set burden across different variant classes. Each panel represent a different variant class. The synonymous variants serve as a control variant class. GRIN2A was removed from all gene-sets to identify other contributing genes. On the $x$ axis, the odds ratios in cases vs controls are given. On the $y$ axis, the names of different gene-sets are given. The red vertical line represents the expected odds ratio of 1 .
The horizontal lines indicate $95 \%$ confidence intervals and are restricted to the maximum of odds ratios over all gene-sets. In that case, points are represented as the points without error bars to their right. The uncorrected $p$-values are shown on top of each point. CADD15 $=$ deleterious predicted missense variants. $\mathrm{LOF}=$ Loss-offunction variants (colour figure online) 
within the combined typical and atypical (RE+ARE) cohort. No other variant-intolerant gene (i.e., being present in the first quartile of RVIS) was significantly enriched for variants in any of the tested patient groups. Although variant enrichment for GRIN2A was not found to be significant after correction for RE and/or ARE separately, the odds ratio for GRIN2A consistently exceeded unity in all the considered datasets (Fig. 1a).

\section{Exome-wide and gene-set burden analysis}

Assuming a shared mutational burden in patients across gene-sets of convergent function and/or pathways, we performed gene-set burden analyses by using the in-house controls. A logistic regression approach was used to account for various confounding variables (see Methods section). No significant exome-wide burden was observed across the different variant classes (Fig. 1b). Despite the fact that none of the gene-sets showed a significant result after multipletesting correction, we found several gene-sets with an odds ratio $>1$ for the CADD15, CADD15+LOF and LOF variant classes, especially for the LOF variants, but not for synonymous variants (Fig. 2). A similar result was seen when we performed the analysis with ARE and RE independently.

\section{Discussion}

We performed the first exome-wide association study investigating rare genetic variants of large effect in 194 patients with childhood focal epilepsies with CTS in comparison with 567 in-house and online available 33,370 population controls from the ExAC database. By performing an unbiased gene-burden analysis of patients against the in-house and ExAC controls (Fig. 1a), we show that, only for GRIN2A rare CADD15, CADD15+LOF and LOF variants are significantly more frequent in $\mathrm{RE}$ and $\mathrm{ARE}$, respectively (odds ratio $>1$ ). Owing to the small sample size and genetic heterogeneity, no other gene or gene-set was significantly enriched for variants after correction for multiple-testing (Fig. 2). Since we observe a consistent trend in the odds ratios for the enrichment of LOF variants in several disease-associated gene-sets, we are optimistic that the availability of larger cohorts in the future can allow to identify other genes associated with RE/ARE.

Acknowledgements We thank all participants and their families for participating in this study. We thank the members of the Genomics Lab and the ERGO support team for their help in sampling the data and in creating the database. Parts of the analysis of this were conducted on the HPC facilities of the University of Luxembourg. The authors are grateful to the study participants, the staff of the Rotterdam
Study and the participating general practitioners and pharmacists. The authors would like to thank the Exome Aggregation Consortium and the groups that provided exome variant data for comparison. A full list of contributing groups can be found at http://exac.broadinstitute.org/a bout. This study makes use of data generated by the DECIPHER community. A full list of centres who contributed to the generation of the data is available from http://decipher.sanger.ac.uk and via email from decipher@sanger.ac.uk.

Author contributions Data analysis: DRB, DL, PM, EMR, HT, KJ, WJ, RK. Supervision of data analysis: PM, RK, HL, BAN. Study design: PN, HL, FZ, BAN, Writing the manuscript: DRB, DL, PM, BAN. Editing and revising the manuscript for scientific content: all authors.

Funding HL, BAN, PN, TS and FZ received grants by the EuroEPINOMICS programme (DFG grant numbers: HL: LE1030/11-1, BAN: BN416/5-1, PN: NU50/8-1, TS: SA434/5-1, FWF grant number: FZ: I643-B09) within the EUROCORES framework of the European Science Foundation (ESF). DRB was supported by the JPND CouragePD research grant. The generation and management of genomics data for the Rotterdam Study are supported by the Netherlands Organisation of Scientific Research NOW Investments (nr. 175.010.2005.011, 91103-012) and the Netherlands Genomics Initiative (NGI)/NOW project nr. 050-060-810 (Netherlands Consortium for Healthy Ageing; NCHA). The Rotterdam Study is funded by Erasmus Medical Center and Erasmus University, Rotterdam, Netherlands Organization for the Health Research and Development (ZonMw), the Research Institute for Diseases in the Elderly (RIDE), the Ministry of Education, Culture and Science, the Ministry for Health, Welfare and Sports, the European Commission (DG XII) and the Municipality of Rotterdam. Funding for the project was provided by the Wellcome Trust.

EuroEPINOMICS consortium contributing members: (contributing centers listed by country) Department of Neurology (Eva M. Reinthaler, Fritz Zimprich) and Department of Pediatrics and Adolescent Medicine, Medical University of Vienna, Vienna (Martha Feucht), 1090 Vienna, Austria; Private Practice for Pediatrics (Hannelore Steinböck), 1150 Vienna, Austria; St. Anna Children's Hospital, Department of Neuropediatrics (Birgit Neophytou), 1090 Vienna, Austria; Department of Pediatrics (Julia Geldner), Hospital SMZ Süd Kaiser-Franz-Josef, 1100 Vienna, Austria; Department of Pediatrics (Ursula Gruber-Sedlmayr), Medical University of Graz, 8036 Graz, Germany; Department of Pediatrics (Edda Haberlandt), Medical University of Innsbruck, 6020 Innsbruck, Austria; Department of Pediatrics (Gabriel M. Ronen), McMaster University, L8N3Z5 Hamilton, Ontario, Canada; Cologne Center for Genomics (Janine Altmüller, Dennis Lal, Peter Nürnberg, Thomas Sander, Holger Thiele) University of Cologne, 50931 Cologne, Germany; Luxembourg Centre for Systems Biomedicine (Roland Krause, Patrick May, Rudi Balling), University of Luxembourg, 4365 Esch-sur-Alzette, Luxembourg; Department of Neurology and Epileptology, Hertie Institute of Clinical Brain Research (Holger Lerche), University of Tübingen, 72076 Tübingen, Germany; Department of Neuropediatrics (Bernd A. Neubauer), University Medical Faculty Giessen and Marburg, 35385 Giessen, Germany.

\section{Compliance with ethical standards}

Conflict of interest The authors declare that they have no conflict of interest.

Ethical approval We confirm that we have read the Journals position on issues involved in ethical publication and affirm that this report is consistent with those guidelines. 


\section{References}

1. Fejerman N. Atypical rolandic epilepsy. Epilepsia. 2009;50 Suppl 7:9-12.

2. Carvill GL, Regan BM, Yendle SC, O'Roak BJ, Lozovaya N, Bruneau N, et al. GRIN2A mutations cause epilepsy-aphasia spectrum disorders. Nat Genet. 2013;45:1073-6.

3. Lemke JR, Lal D, Reinthaler EM, Steiner I, Nothnagel M, Alber $\mathrm{M}$, et al. Mutations in GRIN2A cause idiopathic focal epilepsy with rolandic spikes. Nat Genet. 2013;45:1067-72.

4. Lal D, Reinthaler EM, Altmüller J, Toliat MR, Thiele H, Nürnberg $\mathrm{P}$, et al. RBFOX1 and RBFOX3 mutations in Rolandic epilepsy. PLoS ONE. 2013;8:e73323.

5. Lal D, Reinthaler EM, Schubert J, Muhle H, Riesch E, Kluger G, et al. DEPDC5 mutations in genetic focal epilepsies of childhood. Ann Neurol. 2014;75:788-92. May 1

6. Reinthaler EM, Dejanovic B, Lal D, Semtner M, Merkler Y, Reinhold A, et al. Rare variants in $\gamma$-aminobutyric acid type A receptor genes in rolandic epilepsy and related syndromes. Ann Neurol. 2015;77:972-86.

7. DePristo MA, Banks E, Poplin R, Garimella KV, Maguire JR, Hartl $\mathrm{C}$, et al. A framework for variation discovery and genotyping using next-generation DNA sequencing data. Nat Genet. 2011;43:491-8.

8. De Rubeis S, He X, Goldberg AP, Poultney CS, Samocha K, Ercument Cicek A, et al. Synaptic, transcriptional and chromatin genes disrupted in autism. Nature. 2014;515:209-15.

9. Yang H, Wang K. Genomic variant annotation and prioritization with ANNOVAR and wANNOVAR. Nat Protoc. 2015;10:1556-66.

10. Kircher M, Witten DM, Jain P, O'Roak BJ, Cooper GM, Shendure J. A general framework for estimating the relative pathogenicity of human genetic variants. Nat Genet. 2014;46:310-5. Mar
11. Zhan X, Hu Y, Li B, Abecasis GR, Liu DJ. RVTESTS: an efficient and comprehensive tool for rare variant association analysis using sequence data. Bioinformatics. 2016;32:1423-6.

12. Syrbe S, Hedrich UBS, Riesch E, Djémié T, Müller S, Møller RS, et al. De novo loss- or gain-of-function mutations in KCNA2 cause epileptic encephalopathy. Nat Genet. 2015;47:393-9. Apr

13. Epi4K Consortium, Epilepsy Phenome/Genome Project. Ultrarare genetic variation in common epilepsies: a case-control sequencing study. Lancet Neurol. 2017;16:135-43.

14. Petrovski S, Wang Q, Heinzen EL, Allen AS, Goldstein DB. Genic intolerance to functional variation and the interpretation of personal genomes. PLoS Genet. 2013;9:e1003709-e1003709.

15. Pirooznia M, Wang T, Avramopoulos D, Valle D, Thomas G, Huganir RL, et al. SynaptomeDB: an ontology-based knowledgebase for synaptic genes. Bioinformatics. 2012;28:897-9.

16. Kirov G, Pocklington AJ, Holmans P, Ivanov D, Ikeda M, Ruderfer D, et al. De novo CNV analysis implicates specific abnormalities of postsynaptic signalling complexes in the pathogenesis of schizophrenia. Mol Psychiatry. 2012;17:142-53.

17. Mo A, Mukamel EA, Davis FP, Luo C, Henry GL, Picard S, et al. Epigenomic signatures of neuronal diversity in the mammalian brain. Neuron. 2015;86:1369-84.

18. Darnell JC, Van Driesche SJ, Zhang C, Hung KYS, Mele A, Fraser CE, et al. FMRP stalls ribosomal translocation on mRNAs linked to synaptic function and autism. Cell . 2011;146:247-61.

19. Samocha KE, Robinson EB, Sanders SJ, Stevens C, Sabo A, McGrath LM, et al. A framework for the interpretation of de novo mutation in human disease. Nat Genet. 2014;46:944-50.

20. Firth HV, Richards SM, Bevan AP, Clayton S, Corpas M, Rajan D, et al. DECIPHER: Database of Chromosomal Imbalance and Phenotype in Humans Using Ensembl Resources. Am J Hum Genet. 2009;84:524-33.

21. EpiPM Consortium. A roadmap for precision medicine in the epilepsies. Lancet Neurol. 2015;14:1219-28.

\section{Affiliations}

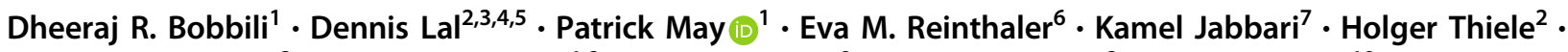
Michael Nothnagel $\left.\right|^{2} \cdot$ Wiktor Jurkowski ${ }^{1,8} \cdot$ Martha Feucht $^{9} \cdot$ Peter Nürnberg $^{2} \cdot$ Holger Lerche $^{10} \cdot$ Fritz Zimprich $^{6}$. Roland Krause $^{1} \cdot$ Bernd A. Neubauer $^{11} \cdot$ Eva M. Reinthaler $^{6} \cdot$ Fritz Zimprich $^{6} \cdot$ Martha Feucht $^{12}$.

Hannelore Steinböck ${ }^{13} \cdot$ Birgit Neophytou $^{14} \cdot$ Julia Geldner $^{15} \cdot$ Ursula Gruber-Sedlmayr $^{16} \cdot$ Edda Haberlandt $^{17}$. Gabriel M. Ronen ${ }^{18} \cdot$ Janine Altmüller ${ }^{2} \cdot$ Dennis Lal ${ }^{2} \cdot$ Peter Nürnberg $^{2} \cdot$ Thomas Sander $^{2} \cdot$ Holger Thiele $^{2}$. Roland Krause $^{1} \cdot$ Patrick May $^{1} \cdot$ Rudi Balling ${ }^{1} \cdot$ Holger Lerche $^{10} \cdot$ Bernd A. Neubauer $^{11}$ EUROEPINOMICS COGIE Consortium

1 Luxembourg Centre for Systems Biomedicine, University of Luxembourg, Esch-sur-Alzette, Luxembourg

2 Cologne Center for Genomics, University of Cologne, Cologne, Germany

3 Psychiatric and Neurodevelopmental Genetics Unit, Massachusetts General Hospital and Harvard Medical School, Boston, MA, USA

4 Program in Medical and Population Genetics, Broad Institute of MIT and Harvard, Cambridge, MA, USA

5 Stanley Center for Psychiatric Research, Broad Institute of MIT and Harvard, Cambridge, MA, USA

6 Department of Neurology, Medical University of Vienna, Vienna, Austria
7 Cologne Biocenter, Institute for Genetics, University of Cologne, Cologne, Germany

8 The Genome Analysis Centre, Norwich, UK

9 Department of Pediatrics, Medical University of Vienna, Vienna, Austria

10 Department of Neurology and Epileptology, Hertie Institute for Clinical Brain Research, University of Tübingen, Tübingen, Germany

11 Department of Neuropediatrics, Medical Faculty University Giessen, Giessen, Germany

12 Department of Pediatrics and Adolescent Medicine, Medical University of Vienna, 1090 Vienna, Austria 
13 Private Practice for Pediatrics, St. Anna Children's Hospital, 1150 Vienna, Austria

14 Department of Neuropediatrics, 1090 Vienna, Austria

15 Department of Pediatrics, Hospital SMZ Süd Kaiser-Franz-Josef, 1100 Vienna, Austria
16

Department of Pediatrics, Medical University of Graz, 8036 Graz, Austria

17 Department of Pediatrics, Medical University of Innsbruck, 6020 Innsbruck, Austria

18 Department of Pediatrics, McMaster University, Hamilton L8N3Z5 ON, Canada 